\title{
La dispersión local de las alturas afecta la inducción tonal
}

\author{
Pablo S. TOledo* E J. Fernando AntA**
}

\begin{abstract}
Resumen
Estudios previos sugerían que la inducción tonal se basa en información acerca de las clases de alturas presentes en una secuencia. Sin embargo, en Anta (2013b) se observó que, aun cuando tal información se conserve constante, la dispersión en el registro de las notas de la secuencia (i.e., la distancia en semitonos que hay entre ellas) afecta la inducción; específicamente, se observó que a mayor distancia entre notas, menor eficiencia en la inducción, y viceversa. El objetivo del presente estudio fue evaluar si los resultados observados en Anta (2013b) se debieron a la variabilidad en la dispersión local de las notas, o en la dispersión global (i.e., a la distancia entre cada nota y la siguiente o entre todas las notas en general, respectivamente). Los análisis aquí realizados sugieren que lo que afectó la inducción tonal fue el incremento de la dispersión local de las notas.
\end{abstract}

Palabras clave: inducción tonal, información sobre las clases de altura, información sobre las alturas, dispersión local de las alturas, dispersión global de las alturas

\section{Title in English: Local pitch dispersion affects tonality induction}

\section{Abstract}

Previous studies suggested that tonality induction is based on the pitch-class related information a sequence shows. However, Anta (2013b) found that, even if that information is kept constant, pitch dispersion (i.e., the distance in pitch/semitones between tones) affects tonality induction; specifically, it was found that the larger the distance between pitches, the weaker the induction, and vice versa. The aim of the present study was to assess whether the results found in Anta (2013b) were due to variability in the local dispersion of pitches, or in their global dispersion (i.e., due to the distance between successive pitches or between all pitches in general, respectively). The analyses conducted here suggest that tonality induction is affected by the local dispersion of pitches.

Keywords: tonality induction, pitch-class related information, pitch-related information, local pitch dispersion, global pitch dispersion

\footnotetext{
* Facultad de Bellas Artes, Universidad Nacional de La Plata (UNLP) - Argentina

E-mail: pablosebastiantoledo@gmail.com

${ }^{* *}$ Facultad de Bellas Artes, Universidad Nacional de La Plata (UNLP); CONICET- Argentina

E-mail: fernandoanta@fba.unlp.edu.ar
} 


\section{Introducción}

Desde la perspectiva del oyente, comprender y apreciar una pieza de música implica la identificación de un sinnúmero de estructuras musicales que permiten organizar y prever el curso de los eventos sonoros. Una de las estructuras a identificar, de especial importancia cuando se trata de piezas arraigadas en la tradición occidental, es la estructura tonal. Esta estructura refiere a las "funciones" o grados de estabilidad perceptual que adquieren los eventos de altura (i.e., notas y acordes) en el contexto musical de referencia: es una estructura jerárquica, en donde unos eventos terminan por ser perceptualmente más importantes (o estables) que otros. Así, los eventos más o menos estables comunican un monto más o menos grande de cierre o "finalización" musical, de conclusión o completamiento, de continuidad o de arribo a un punto de reposo, etc. (Krumhansl, 1990; Lerdahl, 2001).

La estructura tonal de una pieza, el modo en que el oyente comprende sus eventos de altura según grados relativos de estabilidad, depende de un sinnúmero de factores. En términos generales, depende de la interacción de las diferentes estructuras que operan en la pieza (ver, por ejemplo, Lerdahl y Jackendoff, 1983; Lerdahl, 2001). Por ejemplo, la estructura rítmica, con las diferenciaciones entre eventos de mayor o menor duración, o más o menos acentuados, sirve para resaltar cuáles son los eventos más importantes de la estructura tonal; así, una técnica compositiva bastante estandarizada consiste en enfatizar los eventos tonalmente más importantes mediante valores largos o acentos dinámicos, y eclipsar los menos importantes con el uso de valores cortos o sin acento. Por otra parte, lo mismo puede decirse de la estructura formal; en este caso, la técnica consiste en hacer coincidir los puntos de apertura y cierre de los grupos o frases con los eventos que se pretende resulten tonalmente más estables, y viceversa. Así, las estructuras musicales en general se refuerzan, lo que favorece la comprensibilidad de una pieza (al respecto, ver por ejemplo Boltz, 1999).

Ahora bien, en cierta medida, la estructura tonal de una pieza depende específicamente de cómo se organizan las alturas que la componen; esto es, básicamente, depende de qué notas se utilizan y de cómo se las utiliza. Esta puede parecer una apreciación un tanto pueril para el músico profesional. Sin embargo, sus implicancias son sumamente complejas, y aún hoy estimulan y conllevan innumerables esfuerzos de investigación que permitan justipreciar sus alcances. El propósito del presente estudio fue avanzar en esta línea, examinando qué factores relativos a la organización de las alturas musicales facilitan o inhiben el mecanismo inicial de comprensión de la 
estructura tonal: esto es, el mecanismo de inducción de la tonalidad de la pieza considerada. Específicamente, el objetivo fue esclarecer cómo la dispersión de las alturas musicales en el registro incide en la inducción tonal.

\section{Estudios previos sobre inducción tonal: el rol de las cla- ses de alturas musicales}

La inducción tonal es el proceso por el cual el oyente identifica la tonalidad de una pieza o pasaje musical; es decir, el proceso mediante el cual asigna a una nota particular, la tónica, el mayor nivel de estabilidad perceptual, y organiza las notas restantes en niveles de estabilidad decrecientes. Como se anticipó más arriba, la identificación de la tonalidad es el paso inicial hacia la comprensión de la estructura tonal de una pieza, pues implica el reconocimiento de las relaciones "básicas" (i.e., previas a las posibles acentuaciones locales, y a las modulaciones o cambios de tónica) de estabilidad que hay entre las notas. Existe una extensa tradición de estudios orientados a determinar cuáles son los factores (relativos a la organización de las alturas musicales) que facilitan o inhiben la inducción tonal.

Uno de los primeros estudios al respecto es el de LonguetHiggins y Steedman (1971). Según estos autores, el proceso de inducción funciona así: primero, para identificar la tonalidad el oyente procesa las notas entrantes en una secuencia temporal lineal, de "izquierda a derecha"; segundo, con cada nota entrante descarta las tonalidades que no contengan dicha nota en la escala diatónica correspondiente (e.g., la escala de Do mayor, en la tonalidad homónima); y tercero, la inducción termina cuando sólo queda una escala que coincida con las notas disponibles: la escala que resiste el proceso de descarte indica la tonalidad. Si en un determinado momento todas las escalas son eliminadas, se elige aquella cuya tónica o, en su defecto, cuya dominante haya aparecido antes en la secuencia. Esta excepción es propuesta por Longuet-Higgins y Steedman como la "regla de la primera nota", la cual se aplica también si, una vez procesadas todas las notas, quedan dos o más escalas como posibles candidatas. Como el lector podrá notar, este es un modelo bastante simple de inducción tonal que, además, suele arrojar resultados satisfactorios. Por ejemplo, de él se sigue que la tonalidad del fragmento melódico A mostrado en la Figura 1, tomado de la línea de canto del Op. 89-XVI de Schubert, es Mib Mayor, lo cual parece una solución adecuada. 


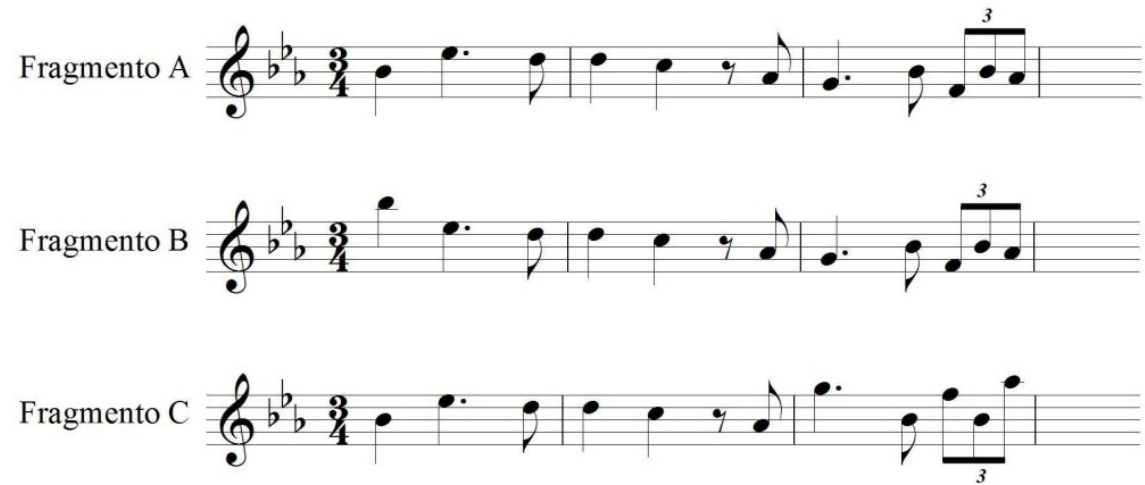

Figura 1. Tres fragmentos melódicos basados en la misma escala, el mismo vector de clases de intervalos, orden, y distribución de clases de alturas. Fragmento A: tomado del Op. 89-XVI de Schubert, y utilizado como estímulo en Autor (2013b). Fragmento B: versión distorsionada de A donde la dispersión local es similar pero la dispersión global aumenta. Fragmento C: versión distorsionada de A donde la dispersión local aumenta pero la dispersión global es similar.

Con todo, el modelo de Longuet-Higgins y Steedman (1971) tiene una estricta dependencia de la noción de "escala", lo que limita su potencial. Efectivamente, para la década de 1980 la idea propia de la Teoría Musical de que "tonalidad" es sinónimo de "escala" había sido absorbida de un modo bastante acrítico por la Psicología de la Música (al respecto, ver Brown y Butler, 1981). El problema de esta idea es que conlleva la presunción de que la escala (como entidad) es la unidad de análisis de la tonalidad. Al respecto, nótese por ejemplo que si se eliminaran las notas lab del Fragmento A de la Figura 1, la "claridad" tonal del mismo se vería más afectada que si se removiese la nota mib, aun cuando mib es la tónica: esto porque, al eliminar el $l a b$, las notas restantes sugieren un mayor número de posibles tonalidades/escalas de referencia; en especial, sugieren la escala de Sib mayor, que por la "regla de la primera nota" pasaría a ser la tonalidad del fragmento. En cambio, al eliminar el mib la única escala de referencia posible es aún la de Mib mayor. Esta situación paradójica nos lleva a la conclusión de que no todas las notas de la escala comunican el mismo monto de información tonal, conclusión que le es ajena al modelo de Longuet-Higgins y Steedman - que toma como unidad de análisis tonal a las escalas, en vez de sus notas. Este tipo de razonamiento llevó a H. Brown y D. Butler a formular un modelo funcional y dinámico de inducción tonal.

Según Brown y Butler (e.g., Brown, 1988; Brown \& Butler, 1981), los oyentes identifican la tonalidad de una pieza a partir de su vector de clases de intervalos, y del orden de sus componentes ${ }^{1}$. El vector

\footnotetext{
${ }^{1}$ El concepto de "clase de intervalo" se funda en el de "clase de alturas". Una clase de alturas es todo grupo de alturas a distancia de 1 o más octavas; dado que las alturas a distancia 
indica cuáles son los intervalos más "raros" (i.e., menos frecuentes) en la pieza, los que, por su rareza, permitirían al oyente "ubicarse en los grados/funciones de la escala" - de allí que el modelo sea "funcional". El orden de las clases de altura que forman los intervalos reforzaría la tonalidad establecida, en tanto enfatice resoluciones típicas (e.g., sensible-tónica, o subdominante-mediante)-de allí que el modelo sea "dinámico". Por ejemplo, a partir del Fragmento A de la Figura 1 el vector de clases de intervalos que se extrae es 2-5-4-3-6-1, lo que significa que a partir de las alturas disponibles pueden inferirse 2 intervalos de $2^{\text {da }}$ menor, 5 de $2^{\text {da }}$ mayor, 4 de $3^{\text {ra }}$ menor, etc. Como muestra el vector, sólo puede inferirse 1 tritono, entre las clases re y lab. El tritono, en un contexto diatónico mayor, siempre se forma entre las notas que "funcionan" como subdominante y sensible: por lo tanto, el tritono re y lab es propio de las tonalidades de Mib mayor y Mib menor (como sensible y subdominante, respectivamente) $\mathrm{o}$, por enarmonía, de las tonalidades La mayor y La menor (como subdominante y sensible, respectivamente). Según el modelo de Brown y Butler, para el oyente estas tonalidades serían las "candidatas" para el fragmento. Ahora, con sólo considerar también la presencia del sib, las tonalidades de La mayor y menor quedan descartadas, pues en estas tonalidades sib es un cromatismo. Finalmente, la presencia del sol-en vez del solb-, indica que el fragmento está en Mib mayor. Esto es, según el modelo de Brown y Butler la tonalidad del fragmento es Mib mayor básicamente debido a la presencia del tritono re-lab, y de las notas sib y sol. La conducción lab-sol, por su parte, reforzaría esa tonalidad, en tanto que replica la dinámica de tensión-reposo propia de la música tonal.

Como lo sugiere este último análisis, el modelo de inducción tonal de Brown y Butler (1981) puede ser igual de efectivo que el de Longuet-Higgins y Steedman (1971). Al mismo tiempo, puede ser teóricamente más adecuado, en tanto atiende a las relaciones internas y temporales que hay entre las notas de una colección, más allá de su adecuación o no a una escala dada. Pero, ¿qué sucedería si en el fragmento analizado en vez de la nota re, la sensible, ocurriera la nota reb, como cromatismo que resuelve en la submediante do? El tritono que se inferiría entonces sería reb-sol, lo cual sugeriría que la tonalidad es Lab mayor. Sin embargo, aun con semejante cambio la melodía parece estar en Mib mayor: ¿tiene sentido este parecer? Según

de octavas se consideran como particularmente "parecidas" o relacionadas entre sí, se dice que pertenecen a la misma clase. Luego, una "clase de intervalo" es la distancia mínima en semitonos que puede medirse entre dos clases de alturas. Por ejemplo, dado el intervalo de $5^{\text {ta }}$ justa descendente sol4-do4, las clases de altura presentes son sol y do, y la clase de intervalo que se forma entre ellas es de $4^{\text {ta }}$ justa, pues la distancia mínima entre las clases se obtiene cuando se recorren el intervalo de sol a do ascendentemente. 
los modelos de Longuet-Higgins y Steedman (1971) y Brown y Butler (1981), no. Sin embargo, sí lo tiene a la luz de los modelos distribucionales de inducción tonal, otro gran cuerpo teórico sobre el tema. Esto es claro si se considera el modelo de Krumhansl-Schmuckler (ver Kurmhansl, 1990), uno de los modelos distribucionales más difundidos (para modelos distribucionales alternativos, ver Temperly, 2007).

El modelo de Krumhansl-Schmuckler postula, primero, que los oyentes son sensibles a cómo se distribuyen las clases de altura en una pieza según su duración total acumulada, de modo que cuanto mayor es esa duración mayor es la estabilidad atribuida a la clase. Segundo, que tras sucesivas audiciones de piezas tonales, los oyentes internalizan las distribuciones de clases de altura que son típicas - las cuales representarían el concepto mismo de tonalidad. La Figura 2 muestra las distribuciones que se asumen como típicas en las piezas en tonalidades mayores y menores; nótese que la clase que se asume con mayor duración es la que haría de tónica. Y tercero, que los oyentes asignan a una pieza la tonalidad (o "distribución típica") que arroja el mayor coeficiente de correlación para con la distribución de clases de altura que la pieza presenta. Por ejemplo, para el Fragmento A mostrado en la Figura 1, cuya distribución de clases de altura también se muestra en la Figura 2, el modelo asigna la tonalidad de Mib mayor, pues el coeficiente más alto se alcanza cuando se correlaciona la distribución (que se asume como) típica del modo mayor con la distribución de la pieza ordenada a partir del Mib.

Ahora bien, de manera interesante, si en el fragmento melódico analizado se cambia la nota re por reb, según se especulaba más arriba, el modelo de Krumhansl-Schmuckler aún predice que los oyentes le asignarán la tonalidad de Mib mayor, pues aún la nota que más duración acumula es mib. Además, incluso con esa modificación se conservan en la distribución de las clases los picos hacia las notas sol y sib, con las que se forma la tríada de tónica (mib-sol-sib). Sin embargo, paradójicamente, si en el fragmento se cambia el mib por el sib (dos notas que prácticamente tienen la misma "importancia" tonal), el modelo predice que su tonalidad será Sol menor, lo cual, ciertamente, no parece una solución adecuada. Como contrapartida, con el cambio de mib por sib tanto el modelo de Longuet-Higgins y Steedman (1971) como el de Brown y Butler (1981) siguen prediciendo que los oyentes escucharán el fragmento en la tonalidad de Mib mayor. 


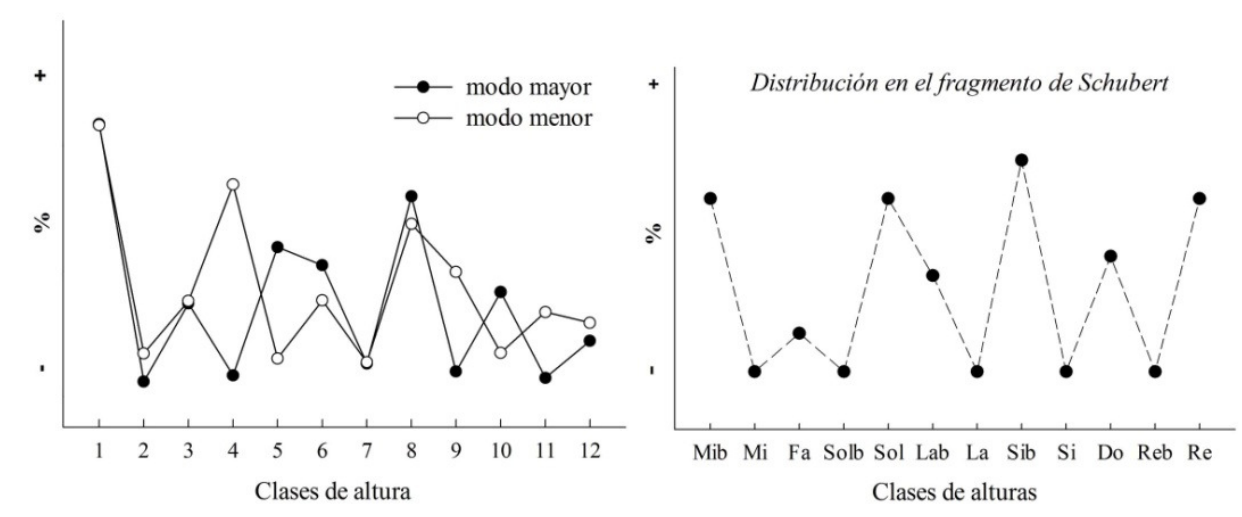

Figura 2. Izquierda: distribuciones de clases de altura típicas de piezas Tonales (después de Krumhansl, 1990); 1= tónica. Derecha: distribución en los fragmetnos de la Figura 1.

Aunque de manera sintética, los ejemplos analizados ilustran cómo operan tres de los modelos de inducción tonal más difundidos en la literatura. Ilustran también cuán "falibles" pueden ser. La identificación de sus falencias nos llevó a analizar qué hipótesis tienen en común que podrían actuar en desmedro de su alcance predictivoexplicativo. La conclusión fue que una hipótesis problemática común es la idea de que las alturas a distancia de octava son perceptualmente equivalentes y que, finalmente, toda altura de una misma clase comunica del mismo modo una función tonal-más allá del contexto en el que se inserte. Al aceptar estas hipótesis como válidas, los tres modelos examinados asumen que la inducción tonal se basa en información acerca de las clases de altura presentes en un pasaje musical dado, independientemente de las alturas efectivamente presentes: en el modelo de Longuet-Higgins y Steedman (1971) lo que importa es si se reconstruye, por ejemplo, la escala de Do mayor, más allá de si el do y el re que aparecen en el pasaje son do4, do5, re3, re2, o cualquier otra nota de estas clases; en el de Brown y Butler (1981), que pueda inferirse el intervalo de tritono o de $2^{\text {da }}$ menor, más allá de que el intervalo presente sea un tritono o una $12^{\text {da }}$, una $2^{\text {da }}$ menor o una $7^{\text {ma }}$ mayor, etc.; en el de Krumhansl y Schmuckler (Krumhansl, 1990), finalmente, si una altura "suma" más duración a una clase u otra, y no qué altura efectivamente agrega esa duración. Como alternativa a este estado del arte, nos planteamos la hipótesis de que la inducción tonal habría de basarse también en información relativa a los intervalos y alturas per se de un pasaje musical. En el apartado que sigue reseñamos brevemente los estudios más importantes que realizamos en relación con esta hipótesis, para luego plantear la pregunta de investigación abordada aquí. 


\section{Estudios propios sobre inducción tonal: el rol de las al- turas musicales}

En nuestra institución hemos realizado una serie de estudios que indican tanto indirecta como directamente que la inducción tonal depende, en parte al menos, de la información disponible acerca de las alturas musicales. Al respecto, dos de esos estudios fueron particularmente importantes, Anta (2013a; 2013b). El primero de ellos, de evidencia indirecta, fue un estudio sobre expectación melódica.

La expectación puede definirse como un complejo proceso, informado tanto por conocimientos previos como circunstanciales de la situación de escucha, mediante el cual el oyente intenta anticipar el curso futuro de los eventos musicales. El objetivo sería estar preparado para asimilar más rápida y eficientemente la información musical entrante, y así regular una respuesta afectiva adecuada (Huron, 2006; Meyer, 1956). En nuestro estudio (Anta, 2013a) examinamos, entre otras, las expectativas tonales de los oyentes; esto es, cuáles notas esperan más dada una tonalidad de referencia. Para ello dimos a escuchar a músicos y no-músicos varios fragmentos de melodías tonales que eran continuados por uno u otro "sonido de prueba", y les pedimos que evalúen cuán bien aquellos fragmentos eran continuados por estos sonidos; el supuesto de esta metodología de pesquisa es que las mejores evaluaciones son para las notas más esperadas, y viceversa. Estudios previos sugerían que, dada la tonalidad del contexto/fragmento, y una vez controlado todo otro factor, las notas de la clase de tónica eran más esperadas que las de las clases de mediante y dominante, que estas eran a su vez más esperadas que las notas de las restantes clases diatónicas, y que la ocurrencia de cualquiera de estas clases era finalmente más esperada que la de las clases cromáticas. Los resultados que obtuvimos no se adecuaron a esta hipotética jerarquía tonal.

Específicamente, nuestros resultados revelaron una interacción entre, por un lado, la distancia registral que separaba a los sonidos de prueba de los del fragmento precedente y, por otro, la hipotética jerarquía/estabilidad tonal de las clases, de modo tal que los juicios de los oyentes respondían a las jerarquías tonales informadas por la literatura sólo cuando los sonidos de prueba estaban próximos en el registro a aquellos presentes en los fragmentos. Por ejemplo, tomando como referencia al Fragmento A de la Figura 1, los resultados indicaron que de las notas de la clase de mediante, sol, sólo el sol4 era considerado por los oyentes como una continuación más adecuada que las notas de la clase supertónica, fa: entre las notas sol3 y fa3, por ejemplo, las diferencias en los juicios de los oyentes no eran significativas. Esta falta de diferencias confiables sucedía también, por ejem- 
plo, entre las notas sol3 y lab3, o entre las notas lab3, sib3, y do4. Las diferencias significativas entre notas (sonidos de prueba) de clases de altura distintas se observaron, básicamente, cuando ellas se ubicaban en el mismo registro que las notas del fragmento. Tomando como referencia al Fragmento A, en el registro de una octava, desde mib4 a mib5.

En suma, al estudio que realizamos sobre expectación melódica sugirió que las relaciones tonales que puede haber entre las notas se debilitaban cuando se las separa en el registro. En lo que se refiere a la indagación acerca de la inducción tonal, ello sugería que el proceso mismo de inducción podía verse debilitado si, además de alejar la nota entrante de las notas precedentes, también estas últimas estaban alejadas unas de otras en el registro. De ser este el caso, debía concluirse que la inducción tonal dependía no sólo de información relativa a las clases de alturas presentes en un pasaje musical dado (i.e., de la escala que sugerían, de las clases de intervalos disponibles, etc.), sino también de información acerca de las alturas que componían al pasaje. (Como el lector podrá notar, esta conclusión es la misma que obtuvimos cuando analizamos qué hipótesis tenían en común los modelos de inducción tonal examinados en el apartado anterior, y cómo esas hipótesis podían actuar en desmedro de su alcance predictivoexplicativo). Restaba entonces examinar cuál era esa información, y testear empíricamente la validez de las nuevas hipótesis. Estas tareas se realizaron en el segundo de nuestros estudios que mencionamos más arriba, el cual proveyó evidencia directa acerca de cómo opera la inducción tonal. Como se verá más abajo, sobre los datos recolectados en este segundo estudio se realizan los análisis aquí presentados. En lo que sigue, entonces, se sintetizan sus resultados más importantes, para plantear las hipótesis aquí examinadas.

En nuestro segundo estudio determinamos inicialmente que cuando se conserva la información disponible acerca de las clases de altura de un pasaje musical pero se modifica alguna de sus alturas, la información disponible acerca de las alturas varía básicamente con respecto a dos factores: la distancia o proximidad registral que hay entre las notas, y su contorno melódico (i.e., su patrón de ascensos y descensos en la dimensión de la altura). Atentos a estos factores, diseñamos dos experimentos con el objetivo de evaluar si uno $u$ otro de ellos incidía en la inducción tonal. En estos experimentos los oyentes debían identificar la tónica de fragmentos de melodías tonales que se presentaban "enteros" o "rotos": esto es, en su estado original, como aparecen en el repertorio, o con sus notas desordenadas en el tiempo, dispersas en el registro, y/o con sus contornos alterados. Es importante notar que, al romper los fragmentos tomados del reperto- 
rio, se conservaban sus escalísticas de referencia, sus vectores de clases de intervalos, y sus distribuciones de clases de altura, pero se eliminaban los patrones de conducción típicos de una melodía tonal (e.g, las resoluciones dominante-tónica de $4^{\text {ta }}$ justa se reemplazaban por saltos de oncena, las resoluciones sensible-tónica de $2^{\text {da }}$ menor ascendente por una $7^{\mathrm{ma}}$ menor descendente, etc.). Sintéticamente, los resultados mostraron que la habilidad de los oyentes de identificar la tónica "correcta" (i.e., la que seleccionaban más para los fragmentos originales) decrecía significativamente de la condición original a la desordenada - lo cual dio un nuevo soporte al modelo de inducción propuesto por Brown y Butler (1981). Sin embargo, mostraron también que tal habilidad decrecía de las condiciones original y desordenada a aquellas condiciones en las que las notas estaban dispersas en el registro, ya sea conservando o no el contorno original. Análisis posteriores indicaron que la merma en la capacidad de identificar la tónica se debía no a las alteraciones del contorno, sino de la distancia registral que separaba a las notas. En síntesis, este estudio informó que aun conservando la información relativa a las clases de altura que contiene un pasaje musical, su estructura tonal se altera si se altera la información que conllevan sus alturas; específicamente, si se incrementa la distancia registral que hay entre ellas.

Ahora bien, de nuestro segundo estudio, específico sobre inducción tonal, surge una interrogante que aún no respondimos adecuadamente: la merma en el acierto en la identificación de la tónica, ¿se debió a que al romper los patrones originales la distancia entre cada nota y la siguiente se incrementó, o a que en general las notas (sucesivas o no) quedaron más distantes entre sí? El problema refiere a la distinción entre lo que podemos denominar dispersión local y dispersión global, respectivamente, de las alturas en el registro. Al respecto, obsérvense los fragmentos A, B y C mostrados en la Figura 1: el Fragmento A fue utilizado como estímulo (en la condición original) en nuestro estudio (Autor, 2013b), mientras que los fragmentos B y $\mathrm{C}$ se agregan aquí para ilustrar el problema a discutir.

Sobre este problema, nótese que el Fragmento B tiene una dispersión local similar a la del Fragmento A $(t(9)=1.00, p>.05)$, porque en uno y otro caso la distancia entre cada nota y la siguiente es similar, pero una dispersión global mayor $(t(54)=3.12, p<.01)$, porque las notas iniciales y finales han quedado muy separadas en el registro. Como contrapartida, el Fragmento $\mathrm{C}$ tiene una dispersión local mayor que el $\mathrm{A}(t(9)=2.33, p<.05)$, debido a los saltos recurrentes, pero una dispersión global similar $(t(54)=1.83, p>.05)$, debido a su registro relativamente estrecho. Este análisis demuestra que es posible alterar la dispersión local de las notas en un contexto dado sin alterar 
su dispersión global, y viceversa; esto es, demuestra que las dispersiones local y global de las alturas pueden operar como dos variables diferentes. En nuestro estudio previo no atendimos a esta dualidad; sólo comparamos el desempeño de los participantes cuando escuchaban las melodías originales, con su desempeño cuando escuchaban melodías dispersas en el registro-independientemente del monto de dispersión. Por lo tanto, no evaluamos en qué medida la merma en la habilidad de los oyentes al identificar la tónica se debía a cambios en una u otra de estas variables de dispersión registral. Evaluar si esa merma se debió a variaciones en la dispersión local o en la dispersión local de los fragmentos utilizados en Anta (2013b) fue el objetivo específico del presente estudio.

\section{Método}

Como se anticipó más arriba, en Anta (2013b) se realizaron dos experimentos. En ambos experimentos, los participantes (35 en cada estudio) debían identificar la tónica de diversos fragmentos melódicos. Los fragmentos eran originales (i.e., tomados del repertorio) y versiones distorsionadas de los mismos, por alteración del orden de las notas y por incremento de su dispersión registral-medida en semitonos-, y/o por alteración de su contorno melódico. La diferencia fue que los participantes del experimento 1 escucharon los fragmentos con el ritmo propio de cada original, mientras que los del experimento 2 escucharon los fragmentos con un ritmo de pulsos constantes. Para el presente estudio se tomaron los porcentajes de acierto promedio obtenidos en la identificación de la tónica en cada uno de los dos experimentos y se planeó un análisis de regresión jerárquica múltiple para cada grupo de datos por separado.

Las variables explicativas generadas para introducir en la regresión fueron cinco. Primero, la variable ORDEN, que codificó el orden de las clases de altura, original o no. Segundo, CONTORNO, en la cual se codificó si los fragmentos presentaban o no el contorno de los originales correspondientes. Tercero, TONICIDAD; esta variable se creó porque un examen preliminar sugirió que unos fragmentos en los que no aparecía la clase de tónica habían promovido, por ese hecho, un porcentaje de acierto menor que los fragmentos restantes. La cuarta variable fue DISPERSIÓN LOCAL; esta variable cuantificó la distancia promedio en semitonos entre cada nota y la siguiente de cada fragmento. Finalmente, la quinta variable fue DISPERSIÓN GLOBAL, la cual cuantificó la distancia promedio en semitonos entre cada nota y todas las otras notas en cada fragmento. 


\section{Resultados}

Para la obtención de resultados, inicialmente las variables predictivas creadas se introdujeron en los análisis de regresión en 3 pasos. En el paso 1 se introdujo ORDEN y CONTORNO, en el paso 2 se introdujo TONICIDAD, y en el 3 se introdujeron simultáneamente las dos variables “objetivo" de este estudio, DISPERSIÓN LOCAL y DISPERSIÓN GLOBAL. Además, en los pasos 1 y 2 el método de regresión fue "introducir", con lo que todas las variables fueron consideradas, mientras que en el paso 3 el método fue "hacia adelante", de modo tal de permitir primero el ingreso en la ecuación de la variable objetivo más eficiente en términos estadísticos, y luego de la variable objetivo restante (pero sólo en el caso de que fuese estadísticamente significativa). La Tabla 1 resume los resultados de estos análisis.

Como puede observarse, los resultados fueron similares para uno y otro experimento. En ningún caso la variable CONTORNO contribuyó de manera significativa a explicar los porcentajes de acierto de los oyentes al identificar la tónica. Este resultado se corresponde con el reportado en Anta (2013b), según el cual las alteraciones del contorno no habían incidido en los porcentajes de acierto de los participantes. Dado que este resultado en Anta (2013b) se obtuvo mediante un ANOVA de medidas repetidas y aquí mediante un análisis de regresión, puede concluirse que, en uno u otro estudio, el mismo no surge de un sesgo asociado a la técnica de análisis utilizada.

Tabla 1

Resumen del ajuste de los modelos de regresión a partir de los porcentajes de acierto en la identificación de la tónica observados en Anta (2013b)

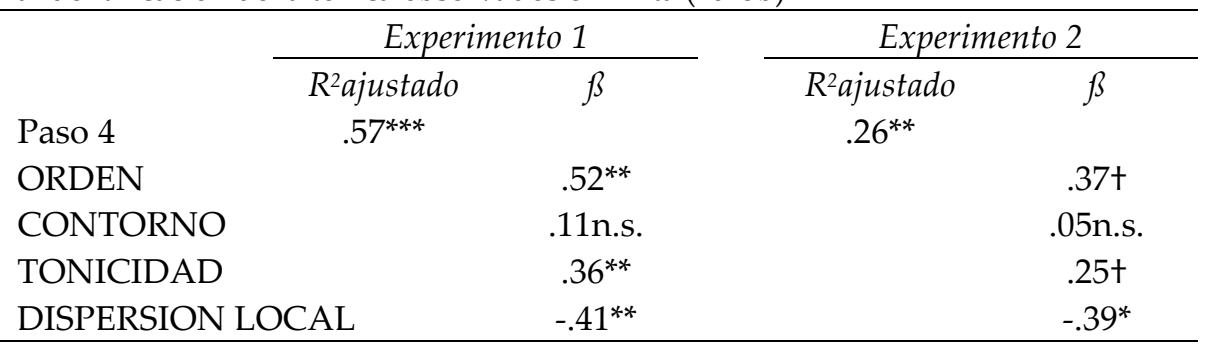

Nota: n.s. $=$ no significativo; $+p<.10 ;{ }^{*} p<.05 ;{ }^{* *} p<.01 ;{ }^{* * *} p<.001$

Para cada experimento, se muestra sólo la variable objetivo (i.e., DISPERSIÓN LOCAL o DISPERSIÓN GLOBAL) incluida en los modelos-i.e., la que resultó estadísticamente significativa.

Por otro lado, la variable ORDEN fue significativa para explicar los datos del Experimento 1, y marginalmente significativa para explicar los datos del Experimento 2. La misma situación se observó con la variable TONICIDAD. Al respecto, es interesante notar que este decremento en la confiabilidad estadística de las variables ORDEN y TONICIDAD ocurre con relación a las respuestas del experimento donde los 
estímulos tenían un ritmo uniforme (i.e., donde al menos gran parte de las pistas temporales de la tonalidad fueron eliminadas). Ello coincide con estudios previos que sugieren que el ritmo juega un rol importante en la percepción de la tonalidad (e.g., Boltz, 1989, 1999). Más importante aún, en lo que a este estudio se refiere, la variable de dispersión que contribuyó a explicar los datos en ambos experimentos fue DISPERSION LOCAL. Al respecto, nótese que el valor de $\beta$ correspondiente a este variable es negativo, lo que indica que los porcentajes de acierto en la identificación de la tónica decrecieron a medida que la dispersión local de las notas en los fragmentos se incrementaba, y viceversa.

Para examinar con más certeza la validez de los resultados sintetizados en la Tabla 1, se llevó a cabo una segunda tanda de análisis de regresión en la cual, ahora, todas las variables explicativas se ingresaron en un único paso y utilizando el método de regresión por pasos. De este modo se evaluó cuáles eran las variables que garantizaban un porcentaje explicativo significativo. Los resultados de estos análisis se resumen en la Tabla 2.

\section{Tabla 2}

Resumen del ajuste de los modelos de regresión por pasos a partir de los porcentajes de acierto en la identificación de la tónica observados en Anta (2013b)

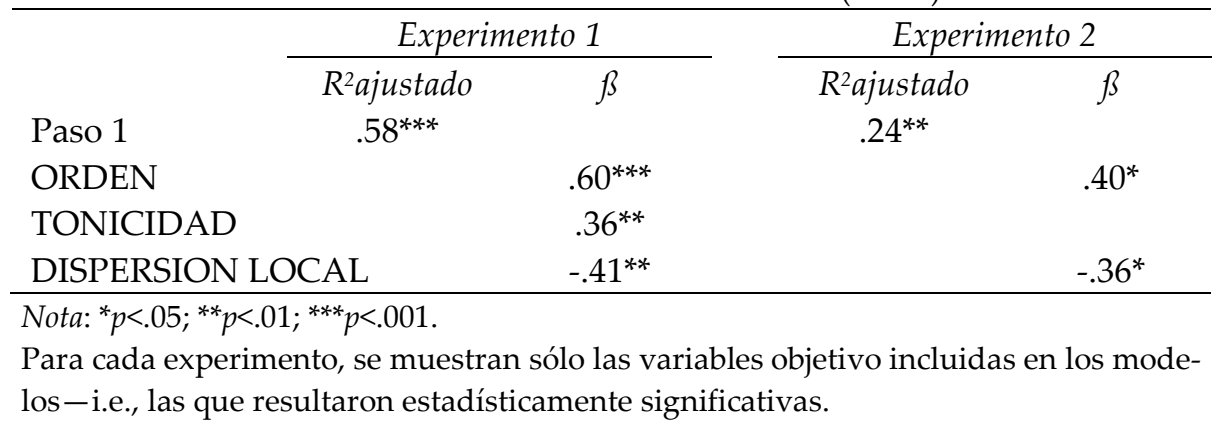

La Tabla 2 muestra que las únicas variables que fueron significativas en ambos experimentos fueron ORDEN y, de manera más importante aún, DISPERSIÓN LOCAL. En el Experimento 2, donde los estímulos utilizados tenían un ritmo uniforme (i.e., donde los ritmos originales fueron removidos), la variable TONICIDAD ya no fue significativa. Esto ratifica la idea sugerida más arriba acerca de la importancia de la información temporal para la inducción tonal. Específicamente, indica que cuando las pistas provistas por las estructuras rítmicas y métricas acerca de la estructura tonal fueron removidas, la presencia o no de clases de altura claves, como puede ser la tónica, perdió relevancia para el proceso de inducción. 


\section{Conclusiones}

En el presente estudio se examinaron tres de los modelos de inducción tonal más difundidos en la literatura: el de Longuet-Higgins y Steedman (1971), el de Brown y Butler (1981), y el de Krumhansl y Schmuckler (ver Krumhansl, 1990). Se argumentó que, más allá de sus virtudes, estos modelos tienen importantes falencias, y que estas se derivan del hecho de que asumen que la inducción tonal depende de las clases de eventos de altura que componen una pieza. Nuestros estudios recientes (Anta, 2013a, 2013b) indicaban que, si bien las hipótesis de los modelos examinados no podían descartarse, para comprender mejor cómo opera la inducción tonal debía tenerse en cuenta también los intervalos y alturas musicales per se de una secuencia, más específicamente, el orden de sus notas y cuán dispersas están en el registro. Acerca de este último factor, sin embargo, no era claro si lo que incidía sobre la inducción tonal era la dispersión local de las alturas de una secuencia, o su dispersión global. Los resultados del presente estudio sugieren que la inducción tonal es afectada por la dispersión local de las alturas, no por la dispersión global.

Desde una perspectiva más bien acotada, los resultados aquí obtenidos sugieren que la comprensión de la estructura tonal de una pieza depende, al menos en parte, de mecanismos de segregación melódica. La segregación melódica, o de "corrientes auditivas" (Bregman, 1990), consiste en agrupar secuencialmente notas próximas en el registro, y generar mentalmente relaciones entre las notas agrupadas. La segregación de líneas melódicas aleatorias al dispersar las notas en el registro, en vez de una línea melódica tonalmente eficiente (con los patrones tonales originales), puede explicar por qué la capacidad de los oyentes de identificar la tónica mermó: la merma se habría debido a que los oyentes conectaron aleatoriamente unas notas con otras, en vez conectar adecuadamente, por caso, la sensible con la tónica, o la subdominante con la mediante, etc. (que en los fragmentos originales siempre aparecían próximas en el registro, pero no así en los fragmentos distorsionados). Dado que, de ocurrir, el mecanismo de segregación opera entre cada nota y la siguiente (i.e., cada nota se agrupa o no a la siguiente, y de cada nota depende que una línea o corriente auditiva tenga continuidad o no), este mecanismo explicaría también por qué la variable que resultó eficiente para explicar las respuestas de los participantes fue DISPERSIÓN LOCAL: la sola dispersión local de las notas determina la naturaleza de la segregación.

Desde una perspectiva más general, finalmente, los resultados sugieren que la inducción tonal es, al menos en parte, un proceso de reconocimiento de patrones (al respecto, ver también Anta, 2013c). 
Como se mencionó más arriba, al cambiar aleatoriamente de registro las notas de una secuencia, como sucedía en los fragmentos utilizados como estímulo en Anta (2013b), se eliminaban sucesiones melódicas típicas de la música tonal. Por ejemplo, un patrón típico de la música tonal es el descenso escalístico por grado conjunto 5-4-3, como dominante-subdominante-mediante, y otro el patrón 8-7-6-5-4-3(2-1), de tónica-a-tónica (Schenker 1906/1990; ver también Salzer, 1952/1995); de hecho, el primero de estos patrones es el que cierra originalmente el Fragmento A mostrado en la Figura 1, y el segundo es el que recorre el fragmento de un extremo a otro. Cuando este fragmento fue aleatoriamente repartido en el registro, uno y otro de estos patrones fueron eliminados, lo cual, ciertamente, puede explicar también por qué la capacidad de los oyentes de identificar la tónica mermó: habría mermado porque los patrones tonales típicos se desintegraron en el registro.

Por último, esta idea de que la inducción tonal es, al menos en parte, un proceso de reconocimiento de patrones, tiene fuertes implicancias no sólo para los estudios en percepción musical, sino también en educación. Sugiere la validez de diversas "reglas" tradicionales en la enseñanza del contrapunto y la armonía (i.e., resolución de sensibles, equilibrio en el registro de las voces, etc.). Además tiene fuertes implicancias para los estudios en análisis y composición musical. Por ejemplo, al sugerir que para que las melodías tonales suenen como tal sus notas deben estar relativamente próximas en el registro, sugiere también que para que las melodías atonales suenan como tal (i.e., para que no permitan la identificación de una tónica) sus notas deben estar dispersas. De manera interesante, disponemos de evidencia preliminar que sugiere la validez de esta presunción Anta (2012). En suma, la evidencia reciente acerca de la incidencia de la dispersión de las alturas en la inducción tonal proyecta un camino fecundo de investigación tanto en el ámbito de la percepción musical, como en las áreas de educación, composición y análisis musical. Estudios posteriores podrían avanzar en una u otra de estas líneas.

\section{Agradecimientos}

Este trabajo fue soportado por el Consejo Nacional de Investigaciones Científicas y Técnicas (CONICET) de Argentina y por subsidios de la Universidad Nacional de La Plata a JFA. 


\section{Referencias}

Anta, J. F. (2012). El repertorio de intervalos utilizados diferencia a la música tonal de la atonal. En Actas de las $6^{0}$ Jornadas de investigación en disciplinas artísticas y proyectuales (JIDAP), Facultad de Bellas Artes, Universidad Nacional de La Plata, Ar.

Anta, J. F. (2013a). Exploring the influence of pitch proximity on listener's melodic expectations. Psychomusicology: Music, Mind, and Brain. En prensa.

Anta, J. F. (2013b). Pitch: a Key Factor in Tonality Induction. En revisión.

Anta, J. F. (2013c). Patrones musicales, esquemas, y metáforas de sentido: Un modelo integral de cognición tonal. Música em Contexto. En prensa.

Boltz, M. G. (1989). Rhythm and "good endings": effects of temporal structure on tonality judgments. Perception \& Psychophysics, 46 (1), 9-17.

Boltz, M. G. (1999). The processing of melodic and temporal information: independent or unified dimensions? Journal of New Music Research, 28 (1), p. 67-79.

Bregman, A. (1990). Auditory scene analysis: The perceptual organization of sound. Cambridge, MA: MIT Press.

Brown, H. (1988). The interplay of set content and temporal context in a functional theory of tonality perception. Music Perception, 5, 219-249.

Brown, H., \& Butler, D. (1981). Diatonic trichords as minimal tonal cuecells. In Theory Only, 5 (6 \& 7), 39-55.

Huron, D. (2006). Sweet anticipation: Music and the psychology of expectation. Cambridge, MA: MIT Press.

Krumhansl, C. L. (1990). Cognitive foundations of musical pitch. New York: Oxford University Press.

110 Lerdahl, F. (2001). Tonal pitch space. New York: Oxford.

Lerdahl, F. \& Jackendoff, R. (1983). A Generative Theory of Tonal Music. MA: MIT Press.

Longuet-Higgins, H. C., \& Steedman, M. J. (1971). On interpreting Bach. Machine Intelligence, 6, 221-241.

Meyer, L. B. (1956). Emotion and meaning in music. Chicago: University of Chicago Press.

Salzer, F. (1952/1995): Audición estructural. Coherencia tonal en música. Barcelona: Labor.

Schenker, H. (1906/1990). Tratado de armonía. Madrid: Real Musical. Temperley, D. (2007). Music and Probability. Cambridge, MA: MIT Press. 